\title{
Design of an E-ELT M1 segment measurement machine with nanometer accuracy
}

\author{
A. Bos ${ }^{a, b}$, R. Henselmans ${ }^{b}$, P.C.J.N. Rosielle ${ }^{a}$, M. Steinbuch ${ }^{a}$, M.J.A. te Voert ${ }^{b}$ \\ ${ }^{a}$ Eindhoven University of Technology, Control System Technology, Department of Mechanical \\ Engineering, Gem-N 0.68, P.O. Box 513, 5600 MB Eindhoven, The Netherlands; \\ ${ }^{b}$ TNO, Opto-mechatronics Department, Stieltjesweg 1, 2628 CK Delft, The Netherlands
}

\begin{abstract}
The baseline design of the European Extremely Large Telescope features a telescope with a 39-meter-class primary mirror (M1), consisting of 798 hexagonal segments. A measurement machine design is presented based on a non-contact single-point scanning technique, capable of measuring the form error of each segment with nanometer uncertainty, fast, and with low operational costs. The implementation of a tactile precision probe eliminates the need for the CMM in the earlier segment manufacturing process. Preliminary assessment show nanometer-level uncertainty after calibration.
\end{abstract}

Keywords: European Extremely Large Telescope (E-ELT), Ground-based astronomy, Giant telescopes, Segment metrology, Measurement machine, Non-contact, NANOMEFOS

\section{INTRODUCTION}

The baseline design of the European Extremely Large Telescope features a telescope with a 39-meter-class elliptical concave primary mirror (M1), consisting of 798 hexagonal segments. Details of the telescope and its requirements can be found in the E-ELT Construction Proposal. ${ }^{1}$ Metrology equipment with nanometer-level uncertainty is required for the final metrology of the mirror segments, this is challenging in view of the required dimensions of around $1.5 \mathrm{~m}$ corner to corner.

This paper describes the preliminary design of a measurement machine for the E-ELT M1 segments. A full length, detailed description of the design can be found in Bos et al. ${ }^{2}$ After an introduction to the E-ELT primary mirror segment assembly and some current metrology methods, a single-point scanning technique is proposed in Sec. 1. The conceptual design of the machine is described in Sec. 2. Section 3 shows the design of the motion system and Sec. 4 explains the design of the metrology system. Finally, an uncertainty estimation of a segment measurement, after calibration, is given in Sec. 5 .

\subsection{E-ELT M1 Segment assembly}

The E-ELT M1 is an elliptical concave mirror with a diameter of approximately 39 meters, an 11-meter central obscuration and a 69-meter radius of curvature (ROC). The M1 mirror consist of discrete optical elements: the primary mirror segments. The segments are near-hexagonal, maximum $1429 \mathrm{~mm}$ in size (corner to corner), 50 mm thick (thickness at the center), and made of low thermal expansion glass. As the primary mirror is not spherical, the hexagonal segmentation pattern has a six-fold symmetry. The segments are grouped in six sectors of 133 segments, thus the primary mirror consists of 798 segments. All 133 segments of a sector are different in shape and optical prescription. ${ }^{1}$

A common support structure is used for the hexagonal segments. Slight counterweight adjustments are needed to compensate for the segment in-plane shape variation between the 133 segment families.

The segment and its whiffle-tree support form a segment assembly, together weighing about $250 \mathrm{~kg}$. The prototype segment assembly as designed by $\mathrm{TNO}^{3-5}$ is depicted in Fig. 1.

The baseline manufacturing plan ${ }^{1}$ of a segment (assembly) is shown in Fig. 2. In phase 2, the support is

Further author information: (Send correspondence to A. Bos.)

A. Bos: E-mail: a.bos@tue.nl, Telephone: +31 402474580

M.J.A. te Voert: E-mail: martijn.tevoert@tno.nl, Telephone: +31 888663119

Advances in Optical and Mechanical Technologies for Telescopes and Instrumentation, edited by

Ramón Navarro, Colin R. Cunningham, Allison A. Barto, Proc. of SPIE Vol. 9151, 91510X

(c) 2014 SPIE · CCC code: 0277-786X/14/\$18 doi: 10.1117/12.2055143

Proc. of SPIE Vol. $915191510 X-1$ 


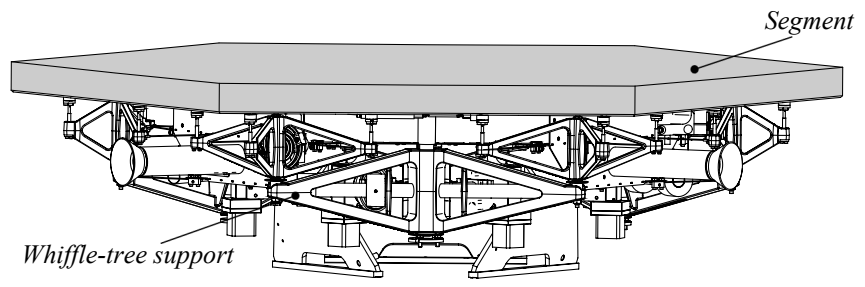

Figure 1. Segment assembly as designed by TNO. ${ }^{3-5}$ A segment assembly consists of a segment and a whiffle-tree support structure.

integrated into the segment, where a coordinate measurement machine (CMM) is used as metrology tool. The measurement machine that is described in this manuscript aims at the last stages of the manufacturing plan, the final metrology step(s), where non-contact metrology is required to prevent damaging the optical surface of the segments. However, by implementing a tactile precision probe in the design of the measurement machine, the need for a separate CMM in the earlier stages of the segment manufacturing process is eliminated.

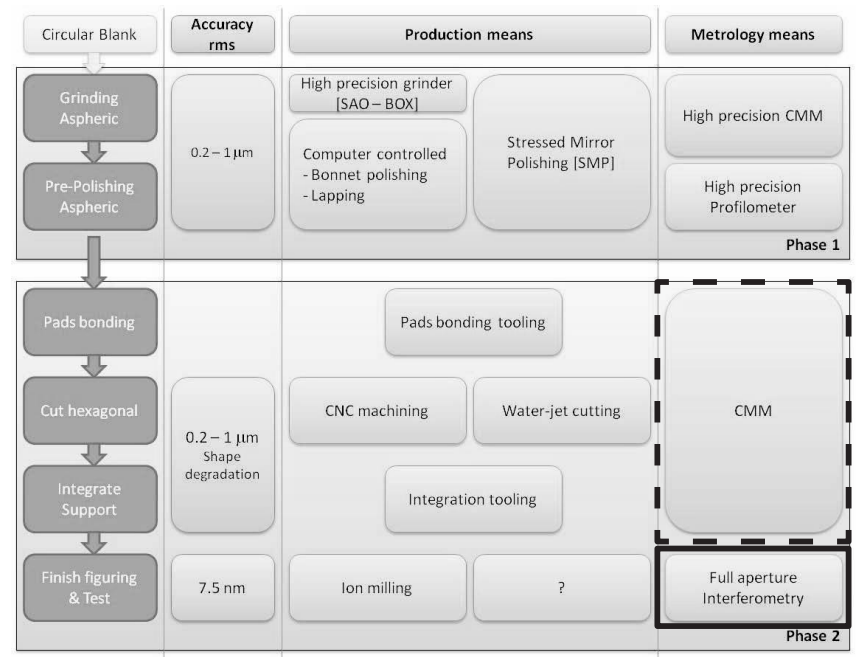

Figure 2. M1 segment assembly manufacturing plan. ${ }^{1}$ The measurement machine aims at the last stages of the manufactrung plan, the final metrology steps of a segment assembly (solid line). By incorporating a tactile precision probe, the $\mathrm{CMM}$ in the earlier manufacturing stage becomes redundant (dashed line).

\subsection{Metrology requirements \& current metrology methods}

All segments cooperate together to form one big M1 mirror having nanometer accuracy. The maximum allowable form error of any segment assembly may not exceed $50 \mathrm{~nm}$ RMS. ${ }^{1}$ After removal of the focus, astigmatism and trefoil ${ }^{6}$ component this error may not exceed $15 \mathrm{~nm}$ RMS. ${ }^{1}$ Based on a TNO assessment, the metrology uncertainty of the form error of the segments (including focus, astigmatism and trefoil) should not exceed $15 \mathrm{~nm}$ RMS and be preferably as low as possible (of course).

Many techniques have been developed to measure mirrors and mirror segments for the existing ground-based telescopes. A successful device will need the capability to measure apertures up to $1500 \mathrm{~mm}$ in diameter at a reasonable speed with nanometer-level uncertainty. Furthermore, this technology must be cost-effective and have low operational cost. All of the techniques and devices that are developed so far have their own disadvantages. One of the most promising techniques is measuring the mirror segments using an imaging technique: Fizeau Interferometry with Computer-Generated Hologram (CGH) correction. ${ }^{7}$ Although measurement times are very short, setup, alignment, and calibration times will be long and thereby increasing cost of operation. Moreover, 
the need for 133 different surface-specific CGHs makes this method non-universal. The predicted accuracy of this method is around $14.4 \mathrm{~nm}$ RMS. ${ }^{7}$ After removing the power and astigmatism component, the measurement errors are stated to be $6.4 \mathrm{~nm}$ RMS.

Another technique, capable of measuring $\varnothing 1.5 \mathrm{~m}$ segments is a swing-arm profilometer. For a swing-arm profilometer, ${ }^{8}$ aligning the rotation axis and the probe to the (non-physical) center of curvature of the surface is difficult, especially because the surface is aspherical. Also, the influence of gravity on the arm is continuously changing, which results in changing forces and thus deflections during measurement. This introduces uncertainty to the position of the probe. Callender et al. ${ }^{9}$ designed their NPL/UCL swing-arm profilometer using a highprecision stage to account for the local distance and slope variations with a wavefront curvature sensor or a Fisba p-phase interferometer. The repeatability they achieve is better than $40 \mathrm{~nm}$. Although the Swing-arm Optical $\mathrm{CMM}$ at the University of Arizona ${ }^{10}$ claims rivalling performance with respect to the Fizeau CGH interferometry test, it is challenging to satisfy the uncertainty requirements due to the increased uncertainty on the position of the probe.

A non-contact profilometer that relies on a moving linear bridge that traverses a probe across the diameter of the optic, measuring the slope across single-line scans, is another alternative. OpTIC Glyndŵr designed an autocollimator-based profiler called OpTIC-NOM ${ }^{11}$ to measure the base radius of curvature of the prototype segments, but it can also be used to determine the form of the segments. OpTIC-NOM is designed to take slope and position data at discrete points and then integrate the data to obtain a height profile across a scanned line. After a scan, the segment can be rotated to measure another line. These lines can be stitched together to obtain a 3D reconstruction of the surface form.

Although it is a simple concept and easy to implement, this method results in long measurement times; it is only possible to scan one line at a time with low velocities, which is time consuming. Therefore, uncertainty due to temperature drifts can be large. Moreover, the limited range of the autocollimator can introduce errors, requiring more stitching steps. Furthermore, for slope measurements, because of the required integration of data, a high-density data set is required. Local surface form checks, by measuring specific points on the surface, are not possible. This could limit the potential of this non-contact profilometer as a metrology tool for mass mirror production.

\subsection{Single-point scanning technique}

As an alternative to the current metrology methods, a measurement machine is proposed based on the principle used in the successful NANOMEFOS ${ }^{12,13}$ (a single-point scanning technique). NANOMEFOS is a fast, noncontact, measurement machine, which is able to measure optics ranging from convex to concave and from flat to freeform, up to $500 \mathrm{~mm}$ diameter, with a measurement uncertainty below $15 \mathrm{~nm}$ RMS (see Fig. 3). The NANOMEFOS has a cylindrical scanning setup with an optical distance probe. The product is mounted on a continuously rotating spindle, while the probe is positioned in radial and vertical direction. Because the probe is oriented perpendicular to the aspherical best-fit of the surface, the sensitivity to tangential errors is reduced, which allows the metrology system to be $2 \mathrm{D}$.

Scaling up the NANOMEFOS would be a logical choice. Nevertheless, the rotating segment concept was rejected for the following reason: the stiffness of the segment assembly is relatively low $\left(10^{7} \mathrm{~N} / \mathrm{m}\right)$ compared to the stiffness that can be obtained in the mechanical design of the machine $\left(10^{8} \mathrm{~N} / \mathrm{m}\right)$. The centripetal force will not only deform the segment support, possibly changing the behavior of the support, but also the segment itself, making it flatter. A preliminary analysis on the effects on the segment assembly rotating with a constant angular velocity was carried out by TNO. At the segment surface, the deformation in the $z$-direction is about 25 $\mathrm{nm}$ PV. The segment will deform about $5 \mu \mathrm{m}$ in radial direction on its whiffle-tree structure. Because the first eigenfrequencies of the segment assembly are around $30 \mathrm{~Hz}$ it is possible that the segment is excited in one of its eigenfrequencies during rotation. This would result in a reduced repeatability of the measurement. In summary, the disturbances acting on the segment during rotation of the segment are not representative compared to its future use in the telescope and also increase the measurement uncertainty.

Therefore, the chosen concept is a more classical orthogonal setup with a translating probe and a stationary segment. This results in a more complex 3D metrology problem. By adding a separate metrology system, this measurement machine, based on a single-point scanning technique, will be capable of measuring large 


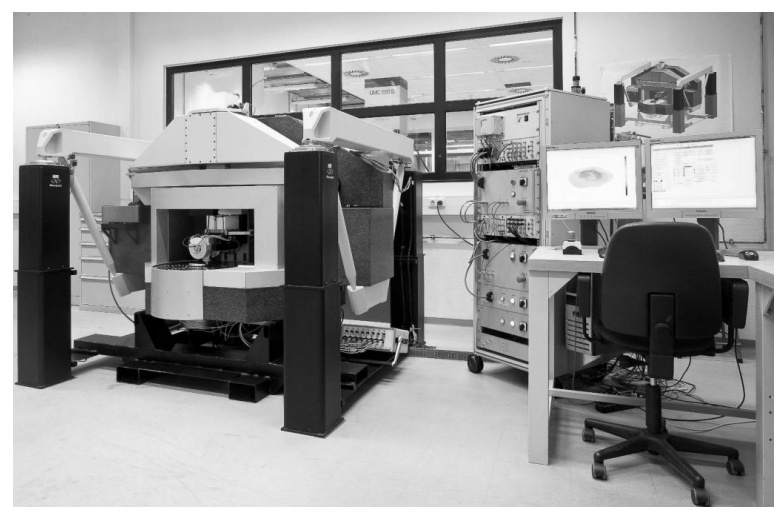

Figure 3. NANOMEFOS, completed machine prototype. ${ }^{12,13}$ This fast, non-contact, machine is able to measure optics ranging from convex to concave and from flat to freeform, up to $500 \mathrm{~mm}$ diameter, with a measurement uncertainty below $15 \mathrm{~nm}$ RMS.

measurement volumes within the required uncertainty. With one or more suitable probe(s), a fast non-contact measurement will be possible. Overall measurement time (including handling of the segment assembly) and cost per measurement are minimized compared to other metrology solutions as alignment effort is reduced, the measurement is fully automated, and no dedicated artifacts for each segment are required. This concept has the following advantages to other principles.

- The typical measurement time of one segment is 15 minutes, using a $3 \mathrm{~mm}$ lateral resolution.

- After the initial calibration, the operational cost of the machine are low, as no dedicated artifacts or CGHs are required in-between measuring consecutive segments.

- Being able to measure all kinds of reference artifacts, the machine has the potential to be traceable to the SI units of length.

- The implementation of a tactile precision probe eliminates the need for the CMM in the earlier manufacturing stages of the segment assembly.

\section{CONCEPTUAL DESIGN}

In the proposed machine design, a non-contact optical probe is translated in $x$ and $y$ (Fig. 4). When a noncontact optical probe with a dynamical range of at least $5 \mathrm{~mm}$ and an acceptance angle of at least $\pm 1^{\circ}$ is used, no vertical translation $(z)$ or probe rotations $(\phi, \psi)$ are necessary, as the maximal sag and slope of a segment is approximately $3.7 \mathrm{~mm}$ and $\pm 0.58^{\circ}$ respectively. This minimises the number of motion axes. The design is

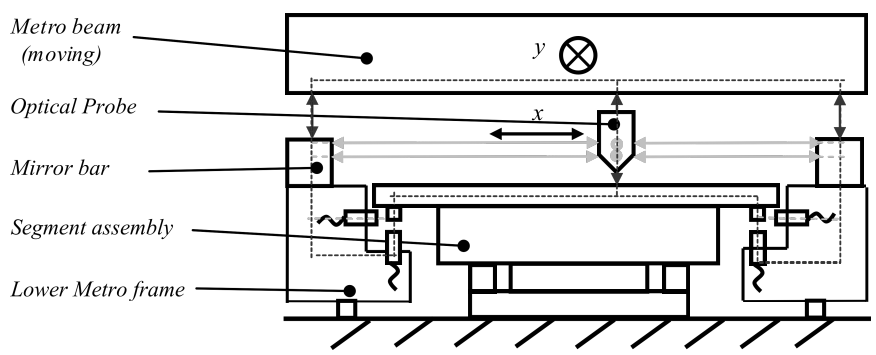

Figure 4. Machine concept: a non-contact optical probe is translated in a plane, using an $x$ - and $y$-translation. The concept is based on a direct and short metrology loop, measured by the metrology system. The metrology beam translates in the $y$-direction. The lower metrology frame acts as a reference for the degrees of freedom of the segment assembly, but also directly for the optical probe in $x, y, \phi$ and $\psi$-direction, and indirectly for the optical probe via the metrology beam in $z$. 
based on a short and direct metrology loop, measured by the metrology system. All degrees of freedom of the segment assembly are measured relative to a lower metrology frame, using capacitive probes measuring on metal blocks bonded to the underside of the segment. The $z$-direction of the non-contact optical probe is measured via a metrology beam to the lower metrology frame using three interferometer axes. Reflective bars are bonded to the Zerodur block of the lower metrology frame. The $x, y, \phi$ and $\psi$ directions of the non-contact optical probe are measured twice relative to these reflective bars using eight interferometer axes, to obtain redundant data and thus reduce the measurement noise (see the 3D metrology setup depicted in Fig. 5).

The basic difference between a standard CMM and this measurement machine is that in this metrology concept all degrees of freedom of the segment are measured relative to a metrology frame, therefore shortening the metrology loop and separating it from the structural loop, reducing the uncertainty. Moreover, the $x, y, z$, $\phi$ and $\psi$-coordinate of the optical non-contact probe are measured directly on the probe, relative to the lower metrology frame.

The machine design is shown in Fig. 6. It measures $3.0 \mathrm{~m}$ wide, $2.6 \mathrm{~m}$ deep and is $1.5 \mathrm{~m}$ high. The total mass is approximately 12.5 tons.

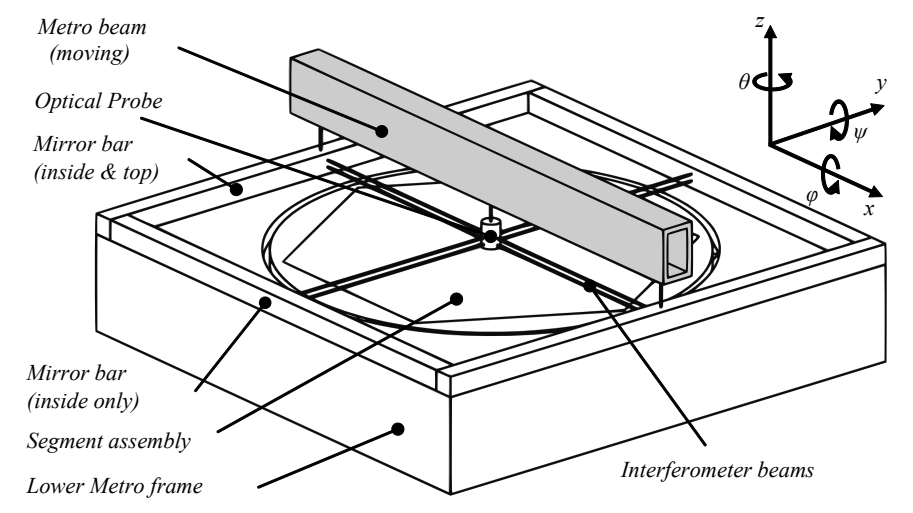

Figure 5. 3D metrology concept. All degrees of freedom of the segment assembly are measured relative to the lower metrology frame. The $z$-direction of the non-contact optical probe is measured via a metrology beam to the lower metrology frame using three interferometer axes. Reflective bars are bonded to the base block of the lower metrology frame. The $x, y, \phi$ and $\psi$ directions of the non-contact optical probe are measured twice relative to these reflective bars using eight interferometer axes, to obtain redundant data and thereby reduce the measurement noise.

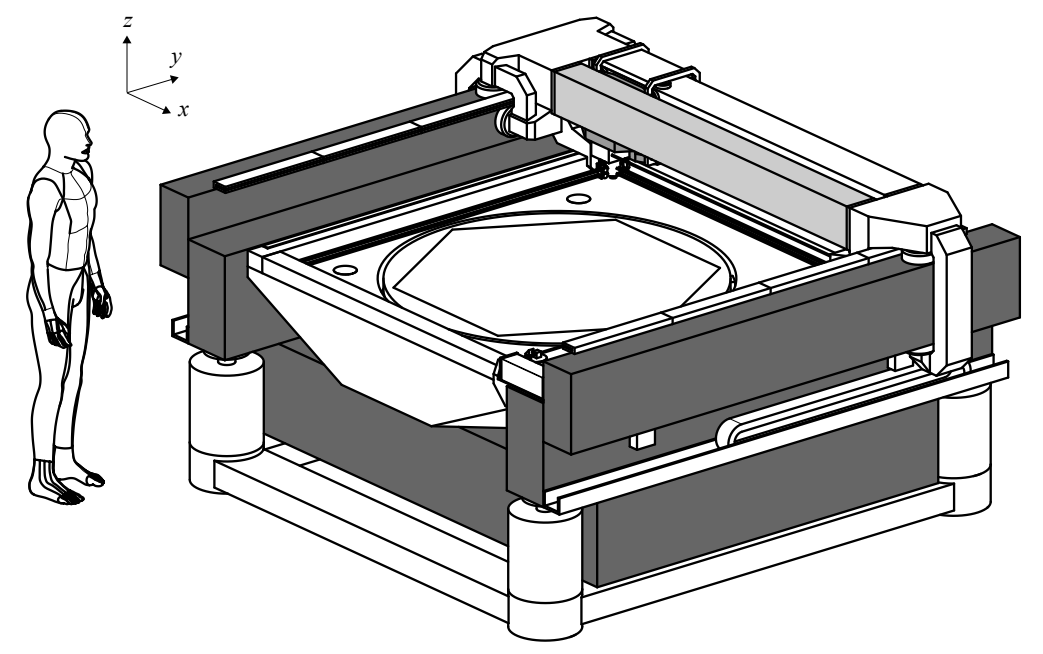

Figure 6. Machine design overview. The machine measures $3.0 \mathrm{~m}$ wide, $2.6 \mathrm{~m}$ deep and is $1.5 \mathrm{~m}$ high. The total mass is approximately 12.5 tons. 
The design consists of two main systems: the motion system and the metrology system. The motion system mainly consists of an $x$-stage and a $y$-stage, which will be discussed in more detail in Sec. 3 . Furthermore, to make fast and repeatable mounting of the segment assembly possible, a segment assembly mounting tool is designed in combination with an extractor mechanism. The mounting tool can be used throughout the manufacturing process of the segment, once its support structure is integrated. The mounting tool has six interfaces to constrain all the degrees of freedom of the segment assembly and it is mounted in a statically determined way on the machine base using three balls and v-grooves. Not three, but six v-grooves are mounted on the base at $60^{\circ}$ each, so it is possible to rotate the segment in steps of $60^{\circ}$, allowing calibration techniques such as the multi-step method.

The metrology system mainly consists of a lower metrology frame, the metrology beam (which is connected to the $y$-stage), the interferometry system, and and optical and tactile probe. These main systems will be discussed in more detail in Sec. 4.

The motion system and the metrology system are both connected to the base, which consists of an assembly of granite blocks, which is supported by four passive vibration isolators. The design has a low center of gravity.

\section{MOTION SYSTEM}

The motion system positions the optical probe relative to the segment in two degrees of freedom. The optical probe is positioned in the measurement plane $(x$ and $y)$ via an $x$-stage and a $y$-stage. The $x$-stage is guided via air-bearings on a guidance beam. The guidance beam is connected to two $y$-carriages guided by air-bearings that together form the $y$-stage. To minimise distortions and hysteresis, the stages have force-closed air-bearing setups with separate position and pre-load frames. Ironless linear motors are applied and high-resolution linear encoders are used for position feedback.

\section{$3.1 x$-stage}

The $x$-stage provides the optical probe with six degrees of freedom. It constrains these such that sub- $\mu \mathrm{m}$ uncertainty is achieved at the probe tip. The $x, y, z, \phi$ and $\psi$-direction are measured by the interferometry system on the optical probe. The $x$-stage is aligned with four air-bearings to a vertical plane of the guidance beam, constraining it in the $y, \phi$ and $\theta$-direction, but, over-constraining it. The position frame to which the bearings are connected has an internal degree of freedom to eliminate this over-constrained direction. Two bearings aligned with the lower horizontal plane constrain the $x$-stage in $z$ and $\psi$-direction. The $x$-direction, which is the direction of motion, is constrained by the linear motor with its servo-stiffness. The maximum stroke that the $x$-stage is capable of is $1510 \mathrm{~mm}$, the mass of the $x$-stage is $15 \mathrm{~kg}$ and it is designed to move with accelerations up to $10 \mathrm{~m} / \mathrm{s}^{2}$.

The $x$-stage front and back view are shown in Fig. 7. The optical probe (1) with optics for the interferometry system is connected to the position frame (2) of the $x$-stage. In this position frame, a hole (3) is machined for weight reduction. In the position frame, an internal degree of freedom (torsion) is introduced to obtain a well-constrained stage. To the position frame, six $\varnothing 65 \mathrm{~mm}$ air-bearings (4) are mounted tilt free. The pre-load frame consists of two stiff frames (5) connected via plates (6). The pre-load bearings are of the piston type, opposing the position bearings. The coil unit of the linear motor (7) is mounted on the position frame, between two bearings.

\section{$3.2 y$-stage}

The $y$-stage moves the probe in the direction perpendicular to the $x$-stage with a low velocity. The guidance beam of the $x$-stage and the metrology beam are part of the $y$-stage and, together with two carriages, form the $y$-stage. In Fig. 8, both the front view (top) and the rear view (bottom) are given. The maximum stroke the $y$-stage is capable of is $1695 \mathrm{~mm}$, the mass of the $y$-stage is $350 \mathrm{~kg}$.

Looking at the front view, the left carriage consists of a position frame (1) with four air-bearings in an orthogonal configuration. The two air-bearings aligned to the top plane (2) constrain the $z$ and $\phi$ motion of the carriage. The two air-bearings aligned to the inside plane of the left granite guidance block (3) are mounted on an intermediate body connected to the position frame (1) via an elastic line-hinge to constrain the motion in $x$-direction only, but with doubled stiffness. These bearings are mounted at the height of the reaction force of the 

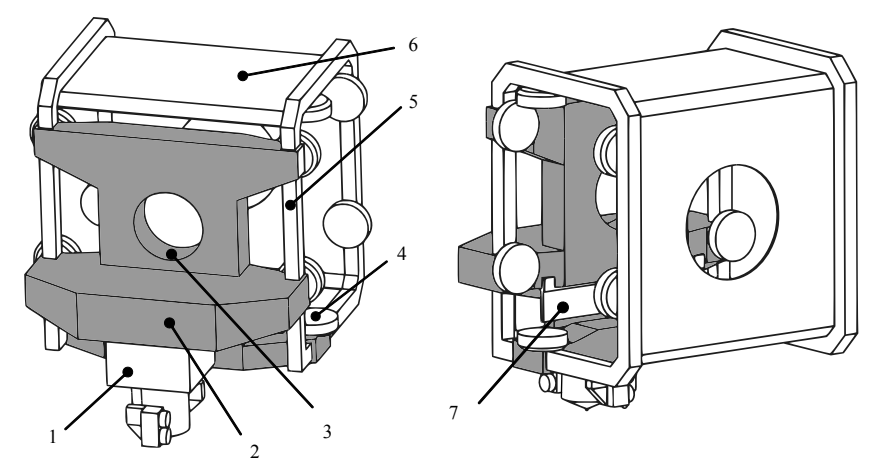

Figure 7. $x$-stage assembly. 1: optical probe, 2: position frame, 3: weight reduction, 4: air-bearings, 5 \& 6: pre-load frame, 7: linear motor.

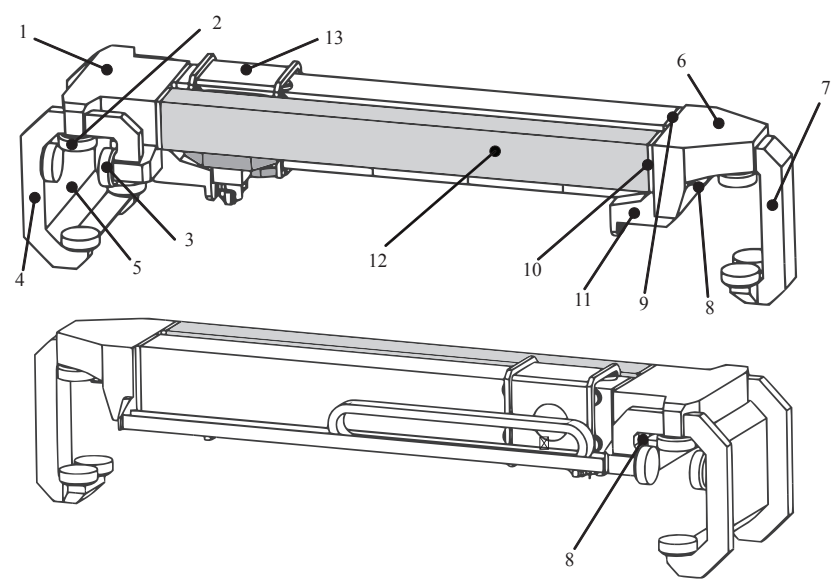

Figure 8. $y$-stage assembly. 1: position frame, $2 \&$ 3: air-bearings, 4 \& 5: pre-load frame, 6: position frame, 7: preload frame, 8: linear motors, 9: guidance beam support interface, 10: metrology beam support interface, 11: linear encoder read-head, 12: metrology beam, 13: $x$-stage.

$x$-stage movement on the guidance beam. The pre-load air-bearings are mounted on two identical stiff frames (4) connected via plates (5), forming the pre-load frame. The right carriage consists of a position frame (6) and a pre-load frame (7) with two air-bearings aligned to the top plane of the guidance block. These air-bearings are mounted tilt free, via cardanic hinges, to an intermediate body. The intermediate body itself is again connected to the position frame (6) via an elastic line-hinge to constrain the $z$-direction only, but with the same $z$-stiffness as the left carriage. The bearings are mounted close to each other to obtain a stiffer intermediate body and to minimise the size of the position and pre-load frame. Coil units of two linear motors (8) are mounted, one each, to the left and right carriage, to constrain the $y$-direction for both carriages. The position frames of the two carriages are mounted on the guidance beam via interfaces (9) bonded to the guidance beam. The metrology beam is also mounted on the position frame of the carriages via intermediate bodies (10), in a statically determined way. Two bodies (11) that house the read head of a linear $y_{1}, y_{2}$ encoder and interferometer optics are mounted, one each, to the $y$-carriages.

The guidance beam is an Alumina $\left(\mathrm{Al}_{2} \mathrm{O}_{3}\right)$ beam with outer dimensions of $300 \times 200 \mathrm{~mm}(\mathrm{H} \mathrm{x} \mathrm{B})$ and a wall thickness of $20 \mathrm{~mm}$. The length of the guidance beam is 2 meters and it weighs $135 \mathrm{~kg}$. The specific stiffness of $\mathrm{Al}_{2} \mathrm{O}_{3}$ is three times higher than that of steel. The coefficient of thermal expansion (CTE) is about $6 \mu \mathrm{m} / \mathrm{m} / \mathrm{K}$. Furthermore, this ceramic tube can be lapped to a straightness in the order of micrometers, making it a good choice for a light and stiff guidance beam with this length. 


\section{METROLOGY SYSTEM}

The metrology system measures the position of the optical probe relative to the segment in all the degrees of freedom using a 12-axis interferometer system, a metrology beam moving in the $y$-direction and a stable lower metrology frame. An exploded view of the metrology system is depicted in Fig. 9. Sintered Silicon Carbide is the material of choice for the metrology beam, due to its excellent thermal and mechanical properties. The lower metrology frame is made of Zerodur, due to its near zero coefficient of thermal expansion. Nanometer-level stabilities are expected.

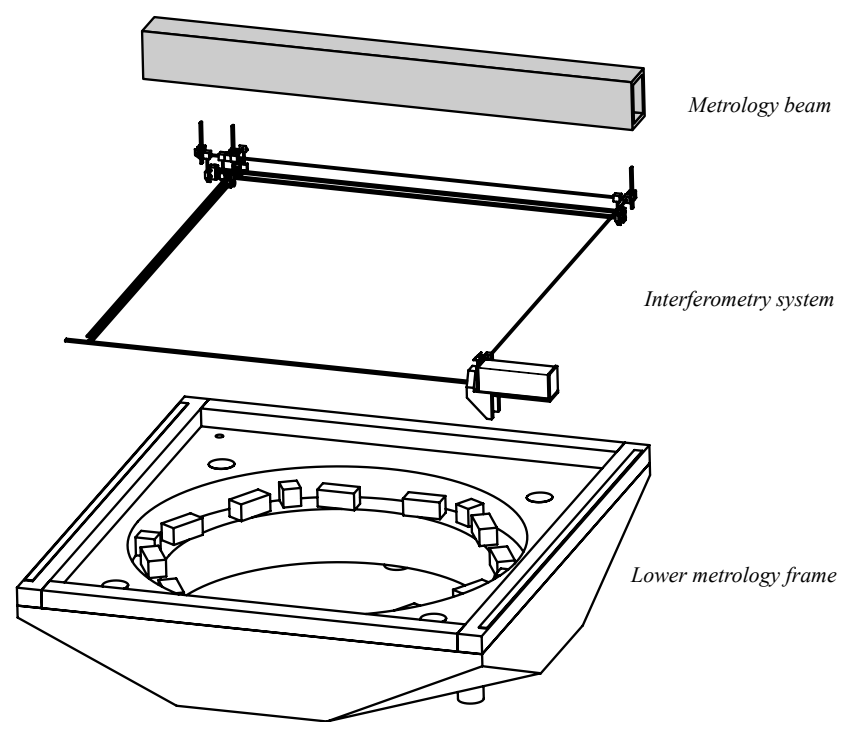

Figure 9. Exploded view of the metrology system. The metrology system is split in a metrology beam (part of the $y$-stage), an interferometry system and a lower metrology frame.

\subsection{Lower metrology frame}

The lower metrology frame is depicted in Fig. 10. The lower metrology frame consists of a block of Zerodur with a hole and chamfered lower corners and part of the edges (1). The hole is machined slightly off-center, as the $y$-stage needs to be moved out of the way to be able to handle the segments. The manufacturing of this Zerodur block could be an issue. To this block, four Zerodur mirrors are bonded with an adhesive. The two bars that act as a reference for the $y$-direction interferometer measurement (2) need to be straight and polished to optical quality on the inside. The two bars that are both a reference for the $x$ - and $z$-direction (3) need to have a straight upper side and inside polished to optical quality. The two Zerodur precision scales (4) that act as a position feedback for the $y$-stage linear motors are bonded on top of the two Zerodur bars by fusion bonding. This is possible because not all of the top surface of these reflective bars is required for the interferometer system. Six volumes where the capacitive probes are placed (5) have to be mounted on the Zerodur block. In this volume, also an adjustment system to put the capacitance sensor in range is required, preferably automated. As an addition, twelve edge sensors (6) can be mounted on the Zerodur frame to obtain information about the real mirror surface positions, as if the segment was positioned in the telescope. As the edge sensors are not on the same position for every segment, these need to be adjustable. Together with the four zero-plane reference mirrors (7), the position and movement of the segment with respect to the metrology frame is known. A sphere (8) is bonded to the Zerodur block to act as an $x, y, z$ nulling-target for the non-contact probe in the home-position. The lower metrology frame is mounted on the granite base block at three points, using three hinged blocks (9).

The mass of the lower metrology frame assembly is $1950 \mathrm{~kg}$ and the first resonance is a torsional mode at $110 \mathrm{~Hz}$. The second resonance is a torsional mode at $160 \mathrm{~Hz}$. 


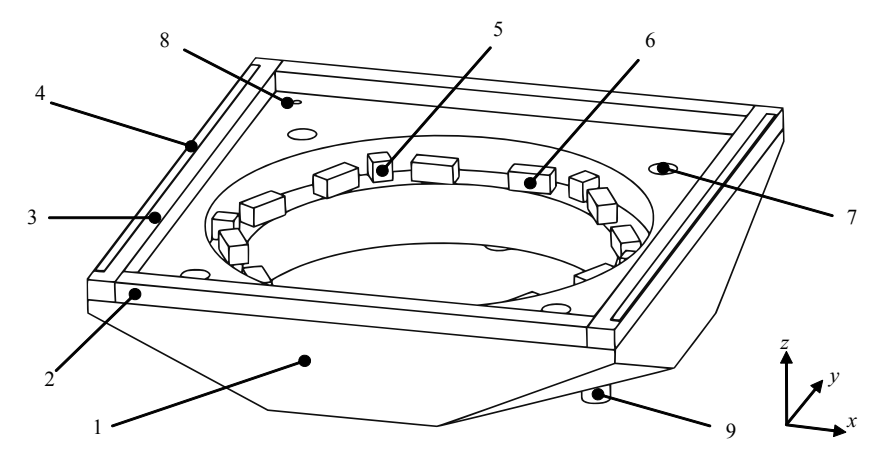

Figure 10. Lower metrology frame assembly. 1: Zerodur block, 2 \& 3: mirror bars, 4: linear encoder precision scales, 5: capacitive probes, 6: edge sensors, 7: zero-plane reference mirrors, 8: nulling-target, 9: hinged support blocks.

\subsection{Metrology beam}

The metrology beam is made of Sintered Silicon Carbide ( $\mathrm{SSiC}$ ). $\mathrm{SSiC}$ has twice the stiffness of steel and only slightly higher density than aluminium. This material has thus a superior specific stiffness. Moreover, its thermal stability is very good as it has a low CTE $(\approx 2 \mu \mathrm{m} / \mathrm{m} / \mathrm{K})$ and a good volumetric thermal diffusivity $\left(84 \cdot 10^{6}\right.$ $\mathrm{m}^{2} / \mathrm{s}$ ), which describes the uniformity and magnitude of the temperature distribution, resulting in a very uniform temperature with a small but present thermal expansion. SSiC is an exotic material and very hard and brittle, difficult to machine, making the price high. The excellent thermal and mechanical properties still make the application of a SSiC metrology beam worthwhile. The material is polishable, which is a great advantage, as the bottom side can be polished to optical quality to act as a reference mirror.

The length of the metrology beam is $2000 \mathrm{~mm}$ and the cross-section is $150 \mathrm{~mm}$ by $200 \mathrm{~mm}$. The wall thickness is the same as the $\mathrm{Al}_{2} \mathrm{O}_{3}$ guidance beam, viz., $20 \mathrm{~mm}$. The mass of the metrology beam is $80 \mathrm{~kg}$.

The metrology beam is mounted on the $y$-stage in a statically determined way to be as thermally and dynamically stable as possible. The first eigenmode at $240 \mathrm{~Hz}$ is bending in the horizontal direction, while the second mode at $310 \mathrm{~Hz}$ is bending in the vertical direction.

\subsection{Interferometry system}

The interferometry system, depicted in Fig. 11, measures the displacement of the optical probe relative to the reference mirror bars on the lower metrology frame. The $x, y, \phi$, and $\psi$ are measured directly, but, depending on the probe position, with a relatively large distance through air (mean value of about $800 \mathrm{~mm}$ in $x$ and 850 $\mathrm{mm}$ in $y$ direction). The $z$ displacement of the optical probe is measured indirectly via a metrology beam to the mirror beams on the lower metrology frame. However, the distance through air is shorter. The $x, y, \phi$ and $\psi$ coordinates are measured twice to get a weighted distance measurement and to obtain redundant data, lowering the measurement noise. This results in eight axes for the interferometer system measuring displacements and tilts. The $z$-measurement of the optical probe to the metrology beam is the ninth axis and adding the two $z$-measurements at the end of the metrology beam to the mirror bars of the lower metrology frame completes the metrology. This results in the need of an eleven-axis interferometry system. Nowadays, twelve-axis interferometer systems $(2 \times 6)$ are commercially available using only one laser. The twelfth interferometer axis can be used to measure the relative air-index variations by measuring from one side of the inner-surface of the Zerodur mirror bars to the other. When the measurement of the air-index variation is done compared to a vacuum tube, the measurement becomes absolute. ${ }^{14}$ An alternative is to use a commercial refractometer.

A heterodyne, double-pass, plane interferometer system is used in the design of this machine. Generally, a heterodyne system is less difficult to align and is less sensitive to noise than a homodyne system. Furthermore, going from a single pass to a double pass interferometer setup reduces the alignment sensitivity. ${ }^{15}$

The 12-axis interferometer system (Fig. 11) is divided into two levels: the upper level with the laser source, the optical-beam supply line for all the interferometers (that will be called the aorta) and the $z$-interferometers. The lower level consists of the four (double-)interferometers for the $x, y, \phi$ and $\psi$ measurement and the twelfth 
axis for measuring air-index variations. The laser source, as a heat source, is located on top of the machine without interfering with the machine functionality and with minimal thermal influence on the metrology frame.

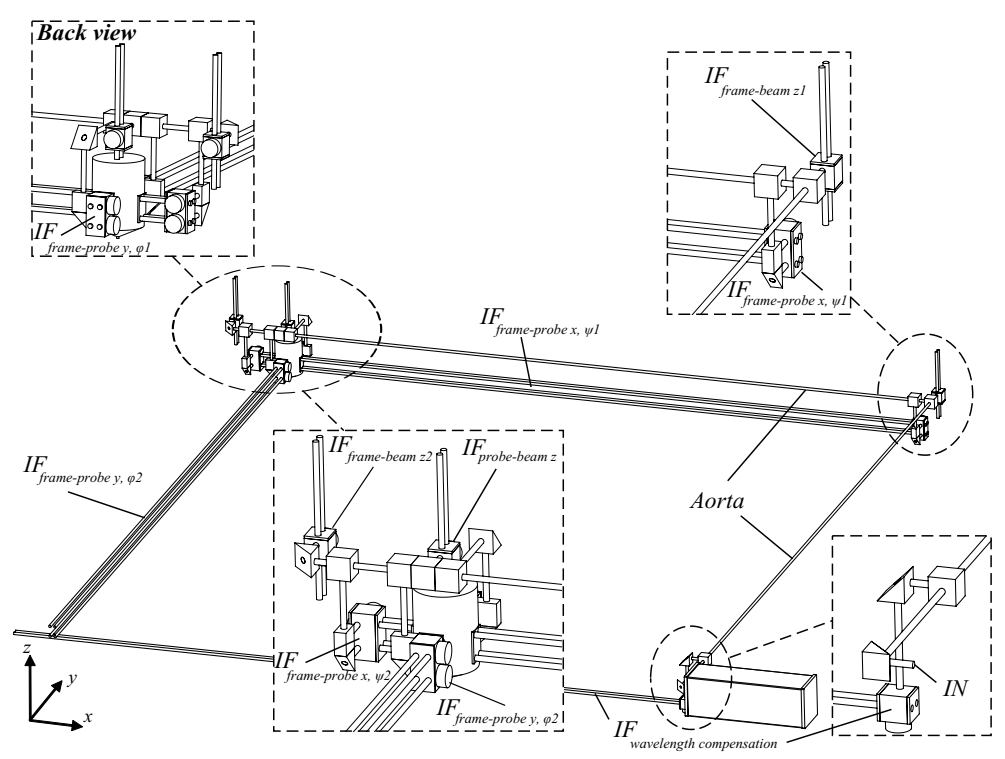

Figure 11. The 12-axis interferometer design. The interferometer system is divided into two levels: the upper level with the laser source, the optical supply line for all the interferometers (the Aorta) and the $z$-interferometers. The lower level consists of four (double-)interferometers for the $x, y, \phi$ and $\psi$ measurement and the twelfth axis for measuring air-index variations.

\subsection{Probes}

A non-contact probe based on the differential confocal principle ${ }^{16}$ is well-suited for the measurement machine. This probe has a $0.1 \mathrm{~nm}$ resolution, an uncertainty of $\leq 10 \mathrm{~nm}$ RMS for medium freeform surfaces $\left(<2^{\circ}\right), 5 \mathrm{~mm}$ measurement range, $\pm 5^{\circ}$ acceptance angle with tilt dependency compensation, and $1.6 \mathrm{~mm}$ distance between lens and segment. The measurement range of $5 \mathrm{~mm}$ is somewhat short, but this can be increased to a safer 8 $10 \mathrm{~mm}$. On the other hand, the acceptance angle of $\pm 5^{\circ}$ could be reduced.

A tactile precision probe is added to the design, mounted next to the optical probe, to be able to measure a ground segment surface with a required ${ }^{1}$ accuracy of $\leq 0.1-0.2 \mu \mathrm{m}$ RMS. As the tactile probe is mounted next to the optical probe, the interferometer system can still be used to obtain information about the $x, y, \phi$, and $\psi$ movement but with a small offset, only resulting in a small error. The tactile probe requires an accurate $z$-guidance with a stroke of about $15 \mathrm{~mm}$.

\section{MEASUREMENT UNCERTAINTY}

The expected measurement uncertainty of the machine after calibration is estimated to be $11.2 \mathrm{~nm}$ RMS. This value is based on a first order uncertainty estimation, experience, ${ }^{12,13}$ knowledge of the designed system, estimates about the accuracies of the principles used, and estimates about the stability of the metrology loop. ${ }^{2}$ It is expected that the individual error sources can be measured using well-known artifacts such as straight-edges, optical flats and true-squares, using self-calibration principles. ${ }^{17}$ Also well-characterized (spherical) mirrors can be used as end-to-end calibration artifacts, together with stitching to cover the entire measurement volume with smaller mirrors. A $1.4 \mathrm{~m}$ spherical reference mirror, which we call the 'master segment', calibrated in an interferometric test tower ${ }^{7,11,18}$ with similar radius of curvature could be used as 'black box' calibration method. Calibrating the artifacts to the required uncertainty level is of course challenging, as reference standards with the required uncertainty over these sizes are not trivial. More research needs to be done on how to calibrate the machine. The machine has the potential to be traceable to the SI unit of length. 
In the design of the machine, redundant information in several directions is available, for instance for the degrees of freedom of the segment by applying twelve capacitive probes, where only six are required as a minimum. This redundant data can be used for averaging, decreasing the measurement noise and increasing the machine's repeatability. Being able to turn the segment assembly in multiple steps of $60^{\circ}$ can decrease the uncertainty, aiding to distinguish several Zernike orders and possible causes of segment deformation due to misalignment and mounting. The measurement strategy could be to measure a coarse grid over the machine before starting a high-resolution measurement, to be able to distinguish thermal drift from segment form.

\section{CONCLUSIONS}

A universal measurement machine design is presented based on principles used in the NANOMEFOS, i.e., a non-contact single-point scanning technique capable of nanometer-accurate form measurements. After the initial calibration, the measurement machine can be used at low operational cost, as no dedicated artifacts or CGHs are required to measure consecutive segments. The typical measurement time of one segment is 15 minutes. In the proposed machine design, the non-contact optical probe is translated in $x$ and $y$. The design is based on a direct and short metrology loop. All degrees of freedom of the segment assembly are measured relative to a Zerodur lower metrology frame. The $z$-direction of the non-contact optical probe is measured via a metrology beam to the lower metrology frame, using three interferometer axes. To this purpose, reflective bars are bonded to the Zerodur base block of the lower metrology frame. The $x, y, \phi$ and $\psi$ directions of the non-contact optical probe are measured relative to these reflective bars, using eight weighted interferometer axes. A 12-axis interferometer system with a single laser source is implemented, allowing the twelfth axis to be used to compensate for air-index variations. A mounting tool is implemented to enable fast and repeatable mounting of the segment assembly in the machine. A central extractor mechanism is designed to lower the segment assembly into the machine, and extract it after measuring. It is possible to rotate the segment assembly in steps of $60^{\circ}$, allowing calibration techniques such as the multi-step method. A tactile precision probe is added to be able to use the machine in earlier stages of the segment manufacturing process, eliminating the need for a separate CMM. Furthermore, reference artifacts and optics with limited slope can be measured to calibrate the machine and potentially make the machine traceable to the SI units of length.

The measurement uncertainty of the machine after calibration is estimated to be $11.2 \mathrm{~nm}$ RMS. This satisfies the estimated uncertainty requirement by TNO of less than $15 \mathrm{~nm}$ RMS.

\section{REFERENCES}

[1] ESO, [The E-ELT Construction Proposal $]$ (2011).

[2] Bos, A., Henselmans, R., Rosielle, P., and Steinbuch, M., "Nanometre-accurate form measurement machine for E-ELT M1 segments," Submitted to Precision Engineering (2013).

[3] Nijenhuis, J., Hamelinck, R., and Braam, B., "Meeting highest performance requirements for lowest price and mass for the M1 segment support unit for E-ELT," Proc. SPIE 7733 (2010).

[4] Nijenhuis, J., Hamelinck, R., and Braam, B., "The optimization of the opto-mechanical performance of the mirror segments for the E-ELT," Proc. SPIE 8336 (2011).

[5] Nijenhuis, J., Hamelinck, R., and Braam, B., "The opto-mechanical performance prediction of thin mirror segments for E-ELT," Proc. SPIE $\mathbf{8 4 5 0}$ (2012).

[6] Zernike, F., "Beugungstheorie des Schneidenverfahrens und Seiner Verbesserten Form, der Phasenkontrastmethode," Physica 1, 689704 (1934).

[7] Burge, J., Zhao, C., and Dubin, M., "Measurement of aspheric mirror segments using Fizeau interferometry with CGH correction," Proc. SPIE 7739 (2010).

[8] Anderson, D. and Burge, J., "Swing-arm profilometry of aspherics," Proc. SPIE 2536 (1995).

[9] Callender, M., Efstathiou, A., King, C., Walker, D., Gee, A., Lewis, A., Oldfield, S., and Steel, R., "A Swing Arm Profilometer for Large Telescope Mirror Element Metrology," Proc. SPIE 6273 (2006).

[10] Su, P., Wang, Y., Oh, C., Parks, R., and Burge, J., "Swing arm optical CMM: self calibration with dual probe shear test," Proc. SPIE 8126 (2011). 
[11] Atkins, C., Mitchell, J., and Rees, P., "Non-contact profilometry of E-ELT segments at OpTIC Glyndŵr," Proc. SPIE 8126 (2011).

[12] Henselmans, R., [Non-contact measurement machine for freeform optics] (2009).

[13] Henselmans, R., Cacace, L., Kramer, G., Rosielle, P., and Steinbuch, M., "The NANOMEFOS non-contact measurement machine for freeform optics," Precision Engineering 35, 607-624 (2011).

[14] Cosijns, S., [Displacement laser interferometry with sub-nanometer uncertainty] (2004).

[15] Bennett, S., "A double-passed Michelson interferometer," Optics Communications 4/6, 428-430 (1972).

[16] Cacace, L., [An Optical Distance Sensor: Tilt robust differential confocal measurement with mm range and nm uncertainty] (2010).

[17] Evans, J., Hocken, R., and Estler, W., "Self-calibration: Reversal, Redundancy, Error Separation, and "Absolute Testing'," Annals of the CIRP 45/2 (1996).

[18] Ruch, E., "Polissage Optique pour les Grands Instruments de la Physique et de l'Astronomie," Presentation, Sagem-REOSC, Bordeaux (2009). 